\title{
Tacrolimus Treatment for Post-COVID-19 Interstitial Lung Disease
}

\author{
Migiwa Ohgushi ${ }^{1}$, Naruhiko Ogo ${ }^{2}$, Toyoshi Yanagihara ${ }^{2}$, Yukiko Harada ${ }^{1}$, Kosuke Sumida $^{1}$, \\ Ayaka Egashira ${ }^{1}$, Tatsuma Asoh ${ }^{1}$, Takashige Maeyama ${ }^{2}$ and Seiji Yoshizawa ${ }^{1}$
}

\begin{abstract}
:
With expansion of the COVID-19 pandemic, reports of post-COVID-19 interstitial lung disease (ILD) have been emerging. However, there are few reports regarding treatment. Some reports indicate that corticosteroids are effective for post-COVID-19 ILD, but the use of long-term corticosteroid carries risks of side effects. We administered tacrolimus to an elderly patient with post-COVID-19 ILD who suffered a respiratory failure relapse during steroid tapering. The respiratory status improved with tacrolimus in the post-acute phase, but pulmonary fibrosis progressed in the late phase. Tacrolimus may be effective for treating post-COVID-19 ILD in the post-acute phase, but it does not halt progression of pulmonary fibrosis.
\end{abstract}

Key words: coronavirus disease 2019, COVID19, interstitial lung disease, tacrolimus, respiratory failure, immunosuppressive agent

(Intern Med 61: 585-589, 2022)

(DOI: 10.2169/internalmedicine.7971-21)

\section{Introduction}

Coronavirus disease 2019 (COVID-19), caused by Severe Acute Respiratory Syndrome Coronavirus 2 (SARS-CoV2), has led to morbidity and mortality at an unprecedented scale globally. Furthermore, there are an increasing number of reports of persistent and prolonged effects after acute COVID19. Nalbandian et al. defined these symptoms as "post-acute COVID-19 syndrome", characterized by symptoms and/or complications developing more than four weeks after the onset of COVID-19 (1).

Post-acute COVID-19 manifests multi-organ abnormalities, including respiratory dysfunction (1). A study from China showed that 6 months after the disease onset, approximately 50\% of COVID-19 patients had at least 1 abnormality on chest $\mathrm{CT}$, the most common of which was ground-glass opacity (GGO) (2). Another study from the US reported that approximately 7\% (59 out of 837) of COVID19 patients initially screened by telephone and followed by chest CT imaging showed persistent post-COVID interstitial changes on chest CT 6 weeks after the onset (3). The majority $(59 \%)$ had an organizing pneumonia (OP) pattern, with the remainder exhibiting symptoms indicating post-COVID19 interstitial lung disease (ILD) (3). While there is growing evidence of post-COVID-19 ILDs, few reports concern their treatment.

We herein report a case of post-COVID-19 ILD that was treated with tacrolimus along with corticosteroids in the post-acute phase.

\section{Case Report}

A 72-year-old man with a medical history of hypertension presented to the outpatient clinic. His main complaints included chills, throat pain, and dysgeusia for 11 days prior to his visit as well as a fever commencing on the day of his visit. He had a 52 pack-year smoking history. He had no obvious history of interstitial lung diseases. His blood pressure was $163 / 95 \mathrm{mmHg}$, pulse $126 / \mathrm{min}$, respiratory rate of $18 /$ min with an $\mathrm{O}_{2}$ saturation of $94 \%$ on a nasal cannula at $3 \mathrm{~L} /$ min. On a laboratory investigation, he had elevated lactate dehydrogenase (480 U/L) and C-reactive protein (CRP) $(20.88 \mathrm{mg} / \mathrm{dL})$ levels. Chest X-ray showed opacities in the bilateral lower lung fields (Fig. 1). Initial chest CT showed diffuse GGO in both lungs and emphysematous changes in

${ }^{1}$ Department of General Medicine, Hamanomachi Hospital, Japan and ${ }^{2}$ Department of Respiratory Medicine, Hamanomachi Hospital, Japan Received: May 17, 2021; Accepted: October 26, 2021; Advance Publication by J-STAGE: December 4, 2021

Correspondence to Dr. Toyoshi Yanagihara, yanagiha@kokyu.med.kyushu-u.ac.jp 


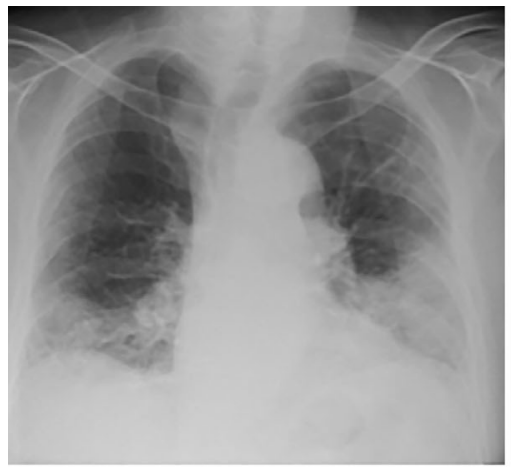

Day 1

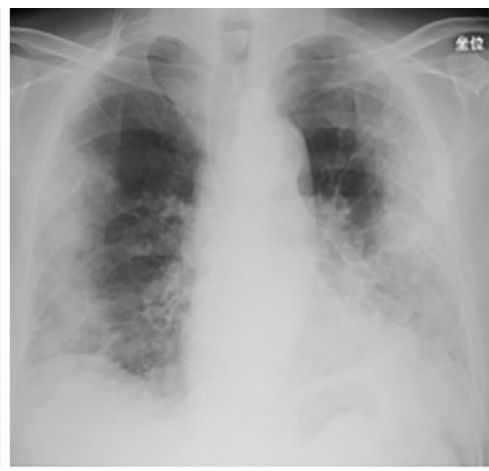

Day 18

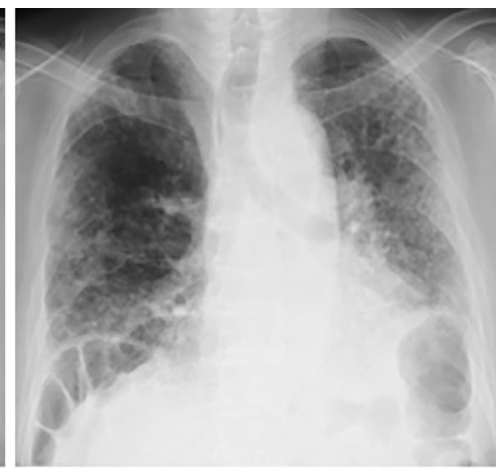

Day 91

Figure 1. Chest X-ray images of the patient. Chest X-ray showed infiltrations in the bilateral lower lung fields at admission (day 1). Bilateral infiltration had progressed by day 18 . By day 91, the findings had mostly improved, but reticular shadows remained with a loss of lung volume.
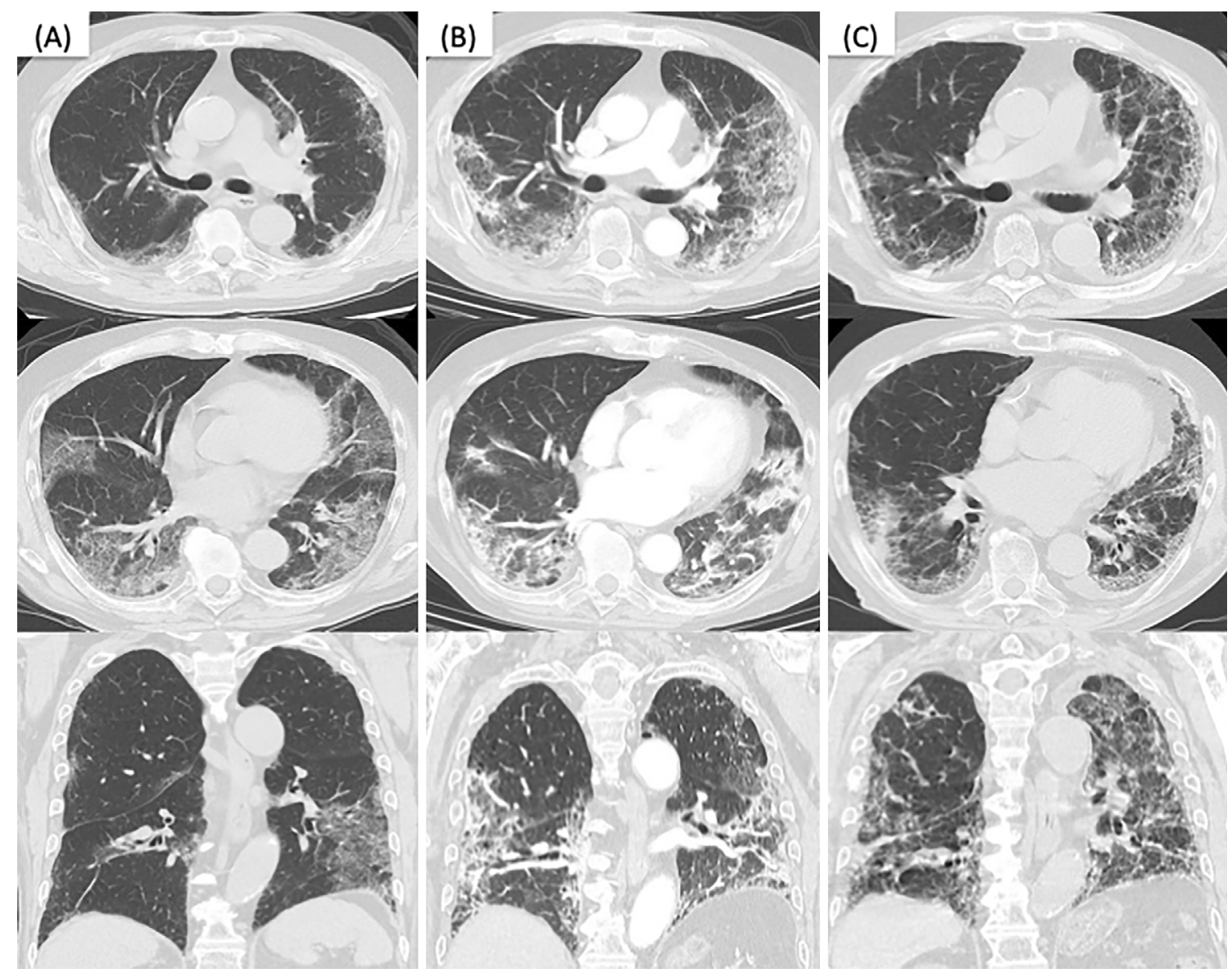

Figure 2. Chest CT images of the patient. (A) Segmental ground-glass opacities (GGOs) were found in the lungs bilaterally on admission. (B) Emerging consolidations and worsening GGOs with some traction bronchiectasis were found on day 18. (C) Consolidations had mostly improved by day 91 at the outpatient clinic, but reticular shadows, worsening traction bronchiectasis, and volume loss were noted.

the background (Fig. 2A). Given his positive polymerase chain reaction (PCR) test for SARS-CoV2, he was diagnosed with COVID-19 and admitted to our department.

Although dexamethasone (6 mg/day) and remdesivir (initial dose of $200 \mathrm{mg} /$ day, followed by $100 \mathrm{mg} /$ day) were initiated, hypoxemia progressed with an oxygen mask at $5 \mathrm{~L} /$ min. The day after admission, methylprednisolone (mPSL) was initiated at $125 \mathrm{mg}$ /day for 3 days, followed by $60 \mathrm{mg} /$ day. On day 8 , after steroid dose reduction, respiratory failure began to progress. A second CT scan of the chest showed progressive, non-segmental GGOs in the bilateral upper lung lobes. Based on these results, a pulsed dose of 


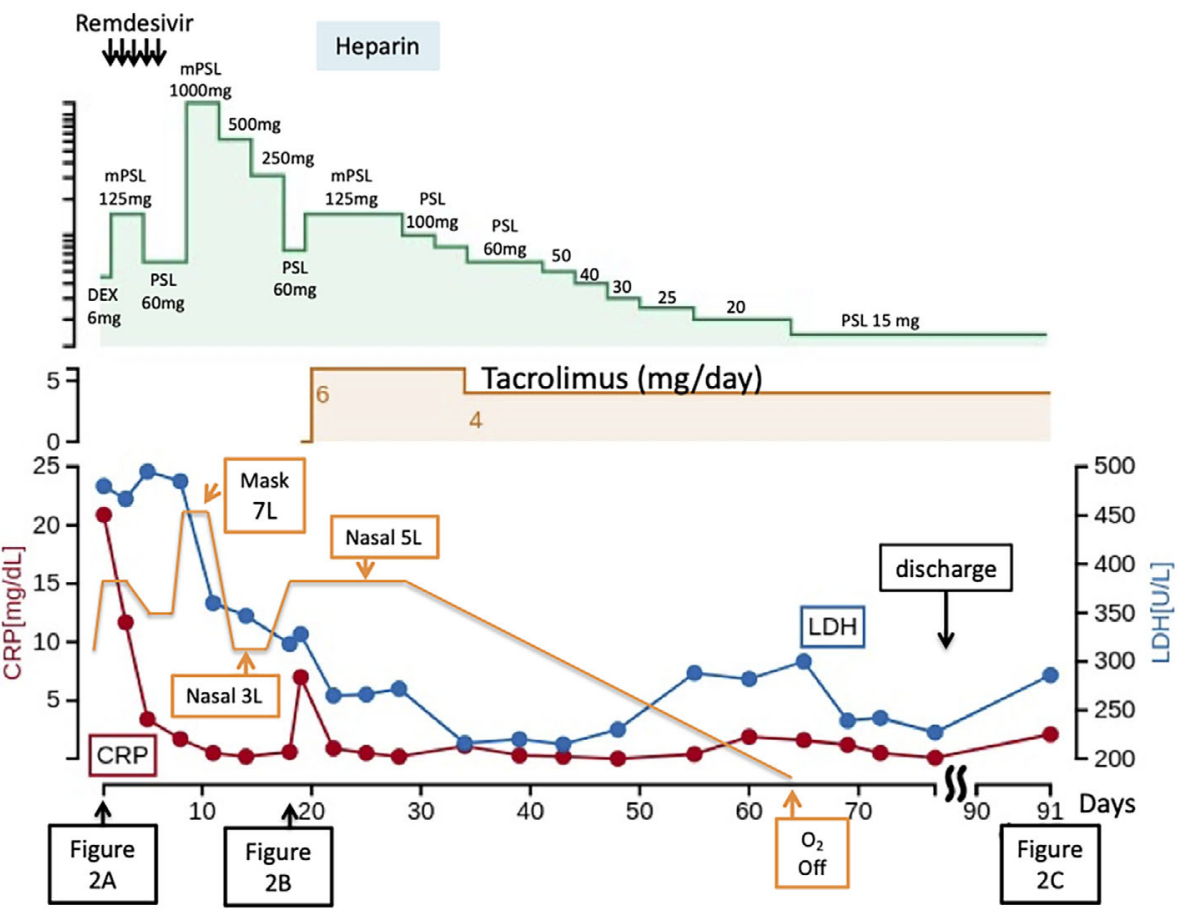

Figure 3. Clinical course of the patient. The patient was treated with dexamethasone $(6 \mathrm{mg} / \mathrm{day})$ and remdesivir, followed by methylprednisolone (mPSL) $125 \mathrm{mg} / \mathrm{day}$. On day 8 , a pulsed dose of mPSL (1,000 mg/day) was started, since respiratory failure had progressed. On day 20, tacrolimus treatment was started. Doses of prednisolone (PSL) were tapered after starting tacrolimus. On day 78, the patient was discharged with $15 \mathrm{mg} / \mathrm{day}$ of PSL and $4 \mathrm{mg} / \mathrm{day}$ of tacrolimus with home oxygen therapy on exertion.

mPSL (1,000 mg/day) was started and tapered every 3 days. Thereafter, the hypoxemia tended to improve (Fig. 3).

On day 18, progressive hypoxemia (on mask at $5 \mathrm{~L} / \mathrm{min}$ ) was observed again when mPSL was reduced to $60 \mathrm{mg} /$ day. Repeated PCR for SARS-CoV2 was negative. A third contrast-enhanced CT scan showed worsening consolidation, GGOs, and traction bronchiectasis, suggesting progressive ILD (Fig. 2B). There was no massive pulmonary thromboembolism. At this point, sputum culture, Aspergillus antigen test, antinuclear antibody, anti-neutrophil cytoplasmic antibody, and rheumatoid factor were all negative. Krebs von den Lungen-6 (KL-6) was elevated to 1,472 U/mL (nor$\mathrm{mal}<500 \mathrm{U} / \mathrm{mL}$ ). Therefore, the $\mathrm{mPSL}$ dose was again increased to $125 \mathrm{mg} /$ day, as $60 \mathrm{mg}$ had proven insufficient to control disease progression.

On day 20, tacrolimus treatment was started, since additional treatment was required to reduce the high dose of corticosteroids for the progressive ILD after COVID-19 pneumonia. Low-molecular-weight heparin (initial dose of 9,600 U/day, followed by 12,000 U/day) was also added for 7 days, considering the thrombotic microangiopathy in pulmonary microvessels, based on the patient's high levels of Ddimer $(12.0 \mu \mathrm{g} / \mathrm{mL})$. On day 56 , his respiratory condition had further improved, and oxygen supplementation was removed at rest. Doses of prednisolone (PSL) were further tapered after starting tacrolimus. Chest X-ray showed improved pulmonary infiltration in general, but GGOs were present in the left upper lung field (Fig. 1). On day 78, he was discharged with $15 \mathrm{mg}$ /day of PSL and $4 \mathrm{mg} /$ day of tacrolimus with home oxygen therapy on exertion. His respiratory status was stable at the outpatient clinic after discharge on day 91. However, follow-up CT showed that lung fibrosis had progressed in some areas (Fig. 2C).

\section{Discussion}

After viral entry, the initial inflammatory response attracts virus-specific $\mathrm{T}$ cells to the infection site and then eliminates infected cells before the virus spreads, leading to recovery in most people (4). In patients who develop severe disease, SARS-CoV2 infection elicits an aberrant host immune response characterized by the overproduction of inflammatory cytokines that accumulate in the lungs $(4,5)$. This hyperinflammation may persist in some patients, further inducing interleukin-6 (IL-6) and transforming growth factor (TGF)- $\beta$ (6), which have been implicated in the development of pulmonary fibrosis. Immunosuppressive therapy is therefore reasonable for hyperinflammatory conditions in persistent COVID-19 (7). Indeed, several reports have shown that corticosteroids are effective for postCOVID-19 ILD $(3,8)$.

In the present case, a pulsed dose of steroids was administered for respiratory failure. A triple-blind, randomized trial revealed that treatment with $\mathrm{mPSL}$ ( $2 \mathrm{mg} / \mathrm{kg} / \mathrm{day})$ reduced the hospital period and mechanical ventilation requirement compared to treatment with $6 \mathrm{mg} /$ day of dex- 
amethasone in cases with hypoxic COVID-19 pneumonia (9). This outcome suggests that the use of high-dose mPSL may be more effective than the standard treatment of $6 \mathrm{mg}$ dexamethasone. However, whether or not the pulsed dose was necessary, considering the side effects, needs to be discussed.

In the present case, the therapeutic effect was achieved with steroid administration, but the condition flared up during steroid tapering, suggesting the need for additional immunosuppression. We were also concerned about side effects associated with long-term steroid activity. In fact, the patient developed severe mediastinal emphysema during the course of the disease, which was thought to be a side effect of steroids. Tacrolimus was then administered in the hope of enhancing steroid efficacy. By confirming the efficacy of tacrolimus by oxygenation level, serum LDH levels, and infiltration areas on chest X-ray, we were able to reduce the steroid dose.

Tacrolimus is an immunosuppressive agent that acts via calcineurin inhibition, mainly on $\mathrm{T}$ cells. It has been used successfully as an immunosuppressant after allogeneic organ transplants to prevent organ rejection $(10,11)$. It has sometimes been used in Japan in concert with corticosteroids for acute exacerbation of ILDs, including idiopathic pulmonary fibrosis (IPF) (12). Given the insufficiency of corticosteroid therapy in the present case, we decided to use tacrolimus. Recently, baricitinib treatment along with remdesivir proved effective in reducing recovery time and accelerating improvement of patients with COVID-19, notably those with severe respiratory failure (13).

Baricitinib, an oral, selective inhibitor of Janus kinases 1 and 2, inhibits the cytokine intracellular signaling pathway that is elevated in severe COVID-19, involving IL-2, IL-6, IL-10, IFN- $\gamma$, and granulocyte-macrophage colonystimulating factor. Artificial intelligence algorithms selected baricitinib as a potential therapeutic for COVID-19 (13). The present findings support the hypothesis that persistent hyper-inflammation may contribute to the pathogenesis of post-COVID-19 ILDs. Based on clinical trials, baricitinib has been approved as a treatment for severe COVID-19 in the US and Japan. Baricitninb would have been considered as a treatment in the present patient, but baricitinib had not yet been approved for treating COVID-19 pneumonia at the time of the diagnosis in this case. Recently, the combination of baricitinib and steroids was reported to be more effective than steroids alone in treating COVID-19 pneumonia (14). Based on these results, we are treating patients with severe COVID-19 pneumonia with a combination of dexamethasone $6 \mathrm{mg}$ or $\mathrm{mPSL} 2 \mathrm{mg} / \mathrm{kg} /$ day, baricitinib, and remdesivir instead of steroid pulse therapy.

A subsequent CT scan performed at the outpatient clinic after discharge showed progression of lung fibrosis in some areas, indicating the efficacy of tacrolimus at the sub-acute stage; however, it was unable to inhibit the progression of fibrosis. As discussed in several papers, anti-fibrotic agents, such as pirfenidone or nintedanib, may be a treatment op- tion for post-COVID-19 pulmonary fibrosis (15). As nintedanib has been recently approved for the treatment of progressive fibrosing-ILD (PF-ILD) in the US, Canada, and Japan, we plan to initiate nintedanib for this patient. The actual efficacy and safety are now being evaluated in a clinical trial (NCT04541680).

In summary, this case demonstrates that immunosuppressive agents, such as tacrolimus with corticosteroids, may be effective for post-acute COVID-19 ILDs but are not sufficient to halt the progression of pulmonary fibrosis. Further studies are warranted to improve available treatments for post-acute COVID-19 ILDs.

Written informed consent was obtained from the patient.

The authors state that they have no Conflict of Interest (COI).

\section{References}

1. Nalbandian A, Sehgal K, Gupta A, et al. Post-acute COVID-19 syndrome. Nat Med 27: 601-615, 2021.

2. Huang C, Huang L, Wang Y, et al. 6-month consequences of COVID-19 in patients discharged from hospital: a cohort study. Lancet 397: 220-232, 2021.

3. Myall KJ, Mukherjee B, Castanheira AM, et al. Persistent postCOVID-19 interstitial lung disease. an observational study of corticosteroid treatment. Ann Am Thorac Soc 18: 799-806, 2021.

4. Mangalmurti N, Hunter CA. Cytokine storms: understanding COVID-19. Immunity 53: 19-25, 2020.

5. Blanco-Melo D, Nilsson-Payant BE, Liu W-C, et al. Imbalanced host response to SARS-CoV-2 drives development of COVID-19. Cell 181: 1036-1045.e9, 2020.

6. McElvaney OJ, McEvoy NL, McElvaney OF, et al. Characterization of the inflammatory response to severe COVID-19 illness. Am J Respir Crit Care Med 202: 812-821, 2020.

7. Mehta P, McAuley DF, Brown M, Sanchez E, Tattersall RS, Manson JJ. COVID-19: consider cytokine storm syndromes and immunosuppression. Lancet 395: 1033-1034, 2020.

8. Vadász I, Husain-Syed F, Dorfmüller P, et al. Severe organising pneumonia following COVID-19. Thorax 76: 201-204, 2020.

9. Ranjbar K, Moghadami M, Mirahmadizadeh A, et al. Methylprednisolone or dexamethasone, which one is superior corticosteroid in the treatment of hospitalized COVID-19 patients: a triple-blinded randomized controlled trial. BMC Infect Dis 21: 337, 2021.

10. Pirsch JD, Miller J, Deierhoi MH, Vincenti F, Filo RS. A comparison of tacrolimus (FK506) and cyclosporine for immunosuppression after cadaveric renal transplantation. Transplantation 63: 977983, 1997.

11. European FK506 Multicentre Liver Study Group. Randomised trial comparing tacrolimus. (FK506) and cyclosporin in prevention of liver allograft rejection. Lancet (London, England) 344: 423-428, 1994.

12. Horita N, Akahane M, Okada $Y$, et al. Tacrolimus and steroid treatment for acute exacerbation of idiopathic pulmonary fibrosis. Intern Med 50: 189-195, 2011.

13. Kalil AC, Patterson TF, Mehta AK, et al. Baricitinib plus remdesivir for hospitalized adults with COVID-19. N Engl J Med 384: 795-807, 2021.

14. Marconi VC, Ramanan AV, de Bono S, et al. Efficacy and safety of baricitinib for the treatment of hospitalised adults with COVID19 (COV-BARRIER): a randomised, double-blind, parallel-group, placebo-controlled phase 3 trial. Lancet Respir Med. Forthcoming.

15. George PM, Wells AU, Jenkins RG. Pulmonary fibrosis and 
COVID-19: the potential role for antifibrotic therapy. Lancet Respir Med 8: 807-815, 2020.
The Internal Medicine is an Open Access journal distributed under the Creative Commons Attribution-NonCommercial-NoDerivatives 4.0 International License. To view the details of this license, please visit (https://creativecommons.org/licenses/ by-nc-nd/4.0/).

(C) 2022 The Japanese Society of Internal Medicine

Intern Med 61: 585-589, 2022 